\title{
The application of differential cloning techniques in post-mortem studies of neuropsychiatric disorders: a way ahead ${ }^{1}$
}

Despite much research, we still have no clear understanding of the exact causes and molecular biology of psychotic illness. Perhaps the clearest contributor to liability to illness is heredity, yet the mode of inheritance and number of genes involved remain undefined. In theory, genetic susceptibility loci may be detected using linkage analysis in multiply affected pedigrees, though uncertainties in diagnosis and genetic parameters make this difficult. Such factors must have contributed to the current disappointments in molecular genetic analysis of psychotic illness, with reports of susceptibility loci either remaining unconfirmed in other pedigrees or being withdrawn by the original authors (Kelsoe et al. 1989; Watt \& Edwards, 1991; Baron et al. 1993). The ultimate reward from genetic studies is at present difficult to predict (Owen, 1992), but is likely to fall well short of the initial expectation that causal genes would be first detectable and then identifiable by pure reverse genetic techniques.

In cases where the genetic component of an illness is complex, it is arguably more rewarding to study the underlying molecular biology of the cell, as this provides a description of the cellular processes occurring during the illness and may also indicate the underlying genetic systems involved. Such an approach has the attraction that it may ultimately provide ways of devising therapeutic management based on cell biological principles.

Gene expression can be studied by the quantitative comparison of mRNA populations in different states. Such an approach can allow the isolation of coordinately controlled genes, and hence may be of great benefit in the psychiatric disorders, which are likely to have a complex genetic basis. In the case of the psychoses a comparison of mRNA extracted from affected brain with that obtained from control, normal brain would allow the identification of genes whose expressions were altered between the two states. More generally, fundamental analysis of gene expression in the brain during development may also be used to determine the molecular mechanisms underlying cell differentiation and organogenesis; such information can provide a 'normal' baseline against which 'affected/diseased' data can be compared in illness states.

As with linkage studies, two approaches are possible for mRNA analysis - the use of candidate genes and the random search for genes whose expressions are changed in the illness. While providing valuable information on the cell biology of illness, candidate gene strategies rarely provide information beyond the researcher's own preconceptions, and have tended to concentrate on a pharmacological description of the illness (e.g. Collinge \& Curtis, 1992). Random search methods, in contrast, are not susceptible to assumptions other than the idea that gene expression may be altered in the neuropsychiatric disorders. Such an approach relies on the identification of genes whose expression is altered in the affected state compared to normal control.

This editorial attempts to explain the basis and prospects for the application of differential and subtractive cloning and screening methodologies in research into the underlying molecular basis of psychotic illness.

\section{THEORETICAL BASIS OF PROTOCOLS}

Differential/subtractive hybridization and cloning techniques allow the isolation of genes whose

1 Address for correspondence: Dr J. Mulcrone, Department of Neuroscience, Institute of Psychiatry, De Crespigny Park, London SE5 8AF. 
relative transcript levels are altered between two RNA populations. Differential screening involves the construction of a cDNA library containing cloned transcripts of genes expressed within a particular tissue or tissues. Subsequent hybridization to cDNA probes synthesized from mRNA extracted from different tissue types under investigation allows the identification of cloned mRNA sequences which are at different abundance levels in the two tissues. Alternatively, two sets of mRNAs from the same tissue under different physiological conditions may be used to identify genes whose expressions are state-dependent. Subtractive hybridization screening is a similar technique which also identifies genes whose expression levels differ in two tissues or different physiological states of the same tissue. In this technique the probe used for screening is enriched in differentially expressed species as a result of the removal of any common sequences by solution hybridization. The pool of differentially expressed sequences generated by this protocol can either be used to screen a cDNA library or can be cloned directly to generate a subtracted library, enriched in differentially expressed sequences, which can then be differentially screened. Once clones demonstrating differential expression are isolated the encoding genes can be identified by sequence analysis. They may be genes which are already identified, or be completely novel.

\section{ANIMAL STUDIES RELEVANT TO BRAIN FUNCTION AND DEVELOPMENT}

A subtractive library approach was used by Porteus et al. (1992) to compare mRNA populations in adult compared to foetal mouse telencephalon in order to determine the processes underlying telencephalon development. This resulted in the isolation of 15 gene sequences (cDNA clones) demonstrating differential expression in the foetal mRNA population, two of which were expressed specifically in differentiating cells. Similarly, the technique has been used to analyse the processes of neural development and differentiation in the rat (Miller et al. 1987), in which cDNA clones were isolated encoding mRNAs with at least tenfold greater abundance in adult compared to foetal brain. mRNA species isolated by these procedures can be further analysed in terms of their spatial and temporal expression patterns by Northern and in situ hybridization analysis. In addition to providing a framework of knowledge, such studies provide molecular markers for cell populations at particular stages of development. The spatial patterns of gene expression in the brain can be analysed by the use of combined subtractive cloning and differential hybridization techniques: e.g. Travis et al. (1987) and Travis \& Sutcliffe (1988) isolated monkey cortex $v$. cerebellum differentially expressed clones which demonstrated spatial specificity by in situ hybridization. Subsequent analysis showed that one of the isolated clones encoded an mRNA whose levels were altered in human Alzheimer disease (AD) frontal cortex tissue compared to normal (Travis \& Sutcliffe, 1988; Bernal et al. 1990). Cell-specific genes can also be isolated using differential screening techniques, e.g. Schmid \& Girou (1987) differentially screened with cerebellum-derived probe a cDNA library constructed from the cholinergic-rich electromotor region of the electric ray. Subsequent analysis of the differential clones identified showed that they hybridized specifically to cholinergic neurons of the electric lobe, and that one encoded an mRNA whose levels increased as a result of electrical stimulation of the region (Schmid, 1991). Therefore, in this example the use of differential screening techniques has resulted in the isolation of an mRNA proposed to be specifically associated with cholinergic synaptic functioning. Similarly, differential screening of a cDNA library constructed from normal mouse cerebellar mRNA with cerebellar mRNA extracted from mutant Purkinje celldegeneration mice resulted in the isolation of three clones encoding mRNAs present in Purkinje cells but either absent from or present at very low levels in other cell types of the cerebellum (Nordquist et al. 1988). Subsequent transgenic manipulation of one of these clones has resulted in the identification of regulatory sequences required for neuronal gene expression (Vandaele et al. 1991). Differential/subtractive hybridization techniques therefore provide a powerful tool in the dissection of the complex molecular processes underlying neuronal development and function. These techniques can also be used to examine the underlying molecular basis of drug, hormone, etc. responses. Subtractive hybridization resulted in the isolation of $\mathrm{mRNAs}$ present in neurons that 
demonstrate selective vulnerability to the neurotoxin trimethyltin (TMT) (Krady et al. 1990), one of which has been shown to encode a peptide, demonstrated by immunocytochemistry, to be specific to TMT-sensitive cells (Toggas et al. 1992). Similarly, eight mRNAs whose expression was induced by nerve growth factor (NGF) have been postulated to be involved in the establishment of neuronal phenotype, following their isolation by the differential screening of cDNA libraries of NGF-treated differentiated cell lines $v$. non-differentiated cells (Leonard et al. 1987). One of these encodes a previously unidentified intermediate filament protein (Leonard et al. 1988). In order to identify proteins involved in blood/brain barrier function Weiler et al. (1990) generated, using subtractive cloning techniques, a pig brain microvessel cDNA library enriched in blood/brain barrier sequences. Subsequent analysis showed that one of the clones encoded apolipoprotein, demonstrated by immunocytochemistry to be present in microvascular endothelial cells.

All these studies served to demonstrate the ability of these techniques to determine the molecular basis of processes occurring in the brain, providing information relevant to brain function and differentiation. Although not of direct relevance to the study of psychotic illness, such studies not only provide knowledge about normal brain function but may also provide candidate genes which can be used to test hypotheses related to illness such as the developmental theory of schizophrenia.

\section{APPLICATION TO HUMAN POST-MORTEM BRAIN STUDIES}

To date, the use of these techniques to study post-mortem brain samples has been limited, primarily as a result of the arguments common to all post-mortem brain studies - potential artefacts caused by post-mortem delay, agonal state, medication and the question as to whether the examination is of 'end stage' phenomena. For example, the subtractive hybridization of a scrapie-infected hamster brain (Duguid et al. 1988) resulted in the isolation of glial fibrillary acidic protein, metallothionein and crystallin mRNAs - all considered to be non-specific markers of neurodegeneration (Walker et al. 1989). However, as with all post-mortem studies the careful choice and preparation of samples in combination with effective data interpretation and prior biochemical and molecular analysis of tissue samples should minimize the above problems. A further argument raised against molecular studies of post-mortem brain tissue is the lability of RNA, since it was generally assumed that the degradative effects of post-mortem delay would render such research unreliable. However, studies have shown that mRNA populations are relatively stable post-mortem (Johnson et al. 1986; Perrett et al. 1992). Northern analysis of mRNA extracted from Alzheimer's disease (AD) brain detected single bands of mRNA encoding amyloid precursor protein and glutamic acid decarboxylase (Walker et al. 1989), indicating limited mRNA degradation. Kobayashi et al. (1990) demonstrated, using cell-specific markers of neuron, oligodendrocyte and astrocyte, no degradation in mRNA up to $12 \mathrm{~h}$ post-mortem. Subsequent construction of cDNA libraries from these mRNA populations demonstrated that they contained full-length transcripts for these marker mRNAs. These authors concluded that post-mortem delay artefacts should not inhibit the use of libraries in the study of altered gene expression in human brain and, as an approach to understanding the molecular mechanisms underlying neuronal degeneration in motor neuron disease, used human brain and spinal cord cDNA libraries to isolate two cDNA clones which were more abundant in spinal cord compared to brain (Kobayashi et al. 1991). A study of mRNA extracted from frontal cortex and hippocampal tissue of AD patients and controls by Ross et al. (1992) showed a very high level of inter-subject variability in the degree of $\mathrm{mRNA}$ and rRNA degradation, although no significant difference between control $v$. AD groups. They found that RNA degradation rates did not correlate with post-mortem delay times, and concluded that hypoxia and ischaemia prior to death may be the primary influence on RNA integrity.

An accepted drawback in the use of differential/subtractive cloning and hybridization techniques is that of the limit of detection - it is generally accepted that mRNAs with an abundance of less than $0.05 \%$ of the total mRNA population will not be detected by this method (Dworkin \& David, 1980). This results in: (a) the inability to detect differentially expressed low-abundance mRNAs, e.g. 
Travis et al. (1987); and $(b)$ the isolation of false negatives, i.e. mRNA species demonstrated to be absent from a particular tissue may be shown by subsequent further analysis to be expressed at low levels. However, the introduction of more sophisticated cloning techniques, and the use of further molecular analysis of isolated clones, circumvents these problems.

Most studies published so far using post-mortem human brain tissue have been in the field of AD research. The differential screening of a human substantia innominata cDNA library with cerebellar probe (Walker et al. 1989) resulted in the isolation of brain-specific substantia innominata-enhanced clones. One of these has been demonstrated to encode a neuron-specific mRNA species whose abundance is $30 \%$ greater in AD temporal cortex compared to normal cortex (Boyes et al. 1992). A monkey cerebral cortex $v$. cerebellum enhanced clone isolated by Travis \& Sutcliffe (1988) was identified as somatostatin I precursor mRNA. Levels of this spatially differentially expressed mRNA were shown to be reduced tenfold in $\mathrm{AD}$ frontal cortex samples. This molecular result correlates with previous immunological findings of the possible involvement of somatostatin in the neuropathology of AD (Davies et al. 1980; Beal et al. 1985). May et al. (1989) examined altered gene expression in AD brain by differential screening of a human hippocampal cDNA library. One of the clones isolated encoded an mRNA with a twofold greater abundance in AD hippocampus compared to normal. Sequence analysis identified the clone as a potential homologue of a rat glycoprotein known to be increased in rat brain following experimental neuronal loss and/or deafferentation. It was proposed that increased expression of the cloned cDNA in AD brain may be a compensatory response to retard the neurodegenerative process (May et al. 1990).

These studies indicate that the generation of cloned cDNAs of interest to regional differentiation has proved useful in the identification of mRNAs differentially expressed between normal and disease tissue. These in turn may prove useful in the understanding not only of the molecular pathology, but also of the genetic basis of illness, even where the complexity of genetic causation tends to confound genetic analysis of families. An example of this is the amyloid precursor protein (APP), which was identified in post-mortem AD brain and characterized subsequently by molecular biological studies. Mutations in the APP gene which are causal in early-onset familial Alzheimer's disease were initially identified despite conflicting evidence from genetic linkage studies (Goate et al. 1991) and subsequently confirmed in small families where linkage analysis alone would not have been feasible. These studies indicate that fairly rare genetic effects may be detected if a good candidate gene is available.

\section{FUTURE APPLICATIONS}

The acceptance that mRNA integrity post-mortem is sufficient to allow the construction of cDNA libraries and their subsequent screening and the fact that these techniques are independent of any prior preconceptions regarding aetiology open a new and possibly lucrative avenue of research into the neuropsychiatric disorders. The results obtained from the use of animal tissue demonstrate the power of these techniques to dissect the molecular basis of cell and regional differentiation in the brain and the effects of drug/hormone treatment. With careful choice and preparation of human brain mRNA samples, the use of differential/subtractive screening techniques should have a role to play in the isolation of novel mRNA species that may be involved in the illness process or in the provision of molecular evidence to support prior biochemical data.

J. MULCRONE AND S. A. WHATLEY

\section{REFERENCES}

Baron, M., Freimer, N. F., Risch, N., Lerer, B., Alexander, J. R., Straub, R. E., Asokan, S., Das, K., Peterson, A., Amos, J., Endicott, J., Ott, J. \& Gilliam, T. C. (1993). Diminished support for linkage between manic depressive illness and X-chromosome markers in three Israeli pedigrees. Nature Genetics 3, 49-54.

Beal, M. F., Mazurek, M. F., Tran, V. T., Chattha, G., Bird, E. D. \&
Martin, J. B. (1985). Reduced numbers of somatostatin receptors in the cerebral cortex in Alzheimer's disease. Science 229, 289-291. Bernal, J., Godbout, M., Hasel, K. W., Travis, G. H. \& Sutcliffe, J. G. (1990). Patterns of cerebral cortex mRNA expression. Journal of Neuroscience Research 27, 153-158.

Boyes, B. E., Douglas, G.W., McGeer, P. L. \& McGeer, E. G. 
(1992). Identification and characterisation of a large human brain gene whose expression is increased in Alzheimer's Disease. Molecular Brain Research 12, 47-57.

Collinge, J. \& Curtis, D. (1991). Decreased hippocampal expression of a glutamate receptor gene in schizophrenia. British Jotrnal of Psychiatry 159, 857-859.

Davies, P., Katyman, R. \& Terry, R. D. (1980). Reduced somatostatin-like immunoreactivity in cerebral cortex from cases of Alzheimer's disease and Alzheimer senile dementia. Nature 288, $279-280$.

Duguid, J. R., Rohwer, R. G. \& Seed, B. (1988). Isolation of cDNAs of scrapie modulated RNAs by subtractive hybridisation of a cDNA library. Proceedings of the National Academy of Sciences, USA 85, 5738-5742.

Dworkin, M. B. \& David, I, B. (1980). Use of a cloned library for the study of abundant poly $\left(\mathrm{A}^{+}\right)$RNA during Xenopus laevis development. Developmental Biology 76, 449-464.

Goate, A., Chartier-Harlin, M. C., Mullan, M., Brown, J., Crawford, F., Fidani, L., Giuffia, L., Haynes, A., Irving, N., James, L., Mant, R., Newton, P., Rooke, K., Roques, P., Talbot, C., Pericak-Vance, M., Rores, A., Williamson, R., Rosser, M., Owen, M.\& Hardy, J. (1991). Segregation of a missense mutation in the amyloid precursor protein gene with familial Alzheimer's disease. Nature 349, 704-706.

Johnson, S. S., Morgan, D. G. \& Finch, C. E. (1986). Extensive postmortem stability of RNA from rat and human brain. Journal of Neuroscience Research 16, 267-280.

Kelsoe, J. R., Ginns, E. I., Egeland, J. A., Gerhard, D. S., Goldstein, A. M., Bale, S. J., Pauls, D. L., Long, R. T., Kidd, K. M., Conte, G., Houseman, D. E. \& Paul, S. M. (1989). Re-evaluation of the linkage relationship between chromosome $11 \mathrm{p}$ loci and the gene for bipolar affective disorder in the old order Amish. Nature 342, 238-242.

Kobayashi, H., Sakimura, K., Kuwano, R., Sato, S., Ikuta, F., Takahashi, Y. (1990). Stability of messenger RNA in post-mortem human brains and construction of human brain cDNA libraries. Journal of Mfolecular Neuroscience 2, 29-34.

Kobayashi, H., Takahashi, H., Oyanagi, K., Ikuta, F., Miyatake, T. \& Tsiyi, S. (1991). Construction of spinal cord cDNA library and application for subtractive cloning of spinal cord-specific cDNA's. Journal of Molecular Neuroscience 3, 59-64.

Krady, J. K., Oyler, G. A., Balaban, C. D. \& Billingsley, M. L. (1990). Use of avidin-biotin subtractive hybridisation to characterise mRNA common to neurons destroyed by the selective neurotoxicant trimethyltin. Molecular Brain Research 7, 287-297.

Leonard, D. G., Zif, E. G. \& Greene, L. A. (1987). Identification and characterisation of $m R N A$ 's regulated by nerve growth factor in PCR cells. Molecular Cell Biology 7, 3156-3167.

Leonard, D. G., Gorham, J. D., Cole, P., Greene, L. A. \& Ziff, E. B. (1988). A nerve growth factor-regulated messenger RNA encodes a new intermediate filament protein. Journal of Cell Biology 106, 181-193.

May, P. C., Lampert-Etchells, M., Johnson, S. A., Poirier, J., Masters, J. M. \& Finch, C. E. (1990). Dynamics of gene expression for a hippocampal glycoprotein elevated in Alzheimer's disease and in response to experimental lesions in rats. Neuron 5, 831-839.

May, P. C., Johnson, S. A., Poirier, J., Lampert-Etchells, M. \& Finch, C. E. (1989). Altered gene expression in Alzheimer's disease brain tissue. Canadian Journal of the Neurological Sciences 16 , 473-476.

Miller, F. D., Naus, C. C., Higgins, G. A., Bloom, F. E. \& Milner, R. J. (1987). Developmentally regulated rat brain mRNA's: molecular and anatomical characterisation. Journal of Neuroscience 7, 2433-2444.

Nordquist, D. T., Kozak, C. A. \& Orr, H. T. (1988). cDNA cloning and characterisation of three genes uniquely expressed in cerebellum by Purkinje neurons. Journal of Neuroscience 8, 4780-4789.

Perrett, C. W., Whatley, S. A., Ferrier, I. N. \& Marchbanks, R. M. (1992). Changes in relative levels of brain specific mRNA species associated with schizophrenia and depression. Molecular Brain Research 12, 163-171.

Owen, M. J. (1992). Will schizophrenia become a graveyard for molecular geneticists? Psychological Medicine, 22, 289-293.

Porteus, M. H., Brice, A. E. J., Bulfone, A., Usdin, T. B., Ciaranello, R. D. \& Rubenstein, J. L. R. (1992). Isolation and characterisation of a library of cDNA clones that are preferentially expressed in the embryonic telencephalon. Molecular Brain Research 12, 7-22.

Ross, B. M., Knowler, J. T. \& McCulloch, J. (1992). On the stability of messenger RNA and ribosomal RNA in the brains of control human subjects and patients with Alzheimer's disease. Journal of Neurochemistry 58, 1810-1819.

Schmid, D. W. (1991). Synaptic activity results in increased transcription as detected by the brain-specific probe $\beta E L-48$ in Torpedo marmorata. Molecular Neurobiology 5, 329-332.

Schmid, D. W. \& Girou, C. (1987). Cloning of a cDNA derived from mRNA of the electric lobe of Torpedo marmorata and selection of putative cholinergic specific sequences. Journal of Neurochemistry 48, 307-312.

Toggas, S. M., Krady, J. K. \& Billingsley, M. I. (1992). Molecular neurotoxicology of trimethyltin: identification of stannin, a novel protein expressed in trimethyltin-sensitive cells. Molecular Pharmacology 42, 44-56.

Travis, G. J. \& Sutcliffe, J. G. (1988). Phenol emulsion-enhanced DNA-driven subtractive cDNA cloning: Isolation of low-abundance monkey cortex-specific mRNAs. Proceedings of the National Academy of Sciences, USA, 85, 1696-1700.

Travis, G. H., Naus, C. G., Morrison, J. H., Bloom, F. E. \& Sutcliffe, J. G. (1987). Subtractive cloning of complementary DNA's and analysis of messenger RNAs with regional heterogeneous distributions in primate cortex. Neuropharmacology 26, 845-854.

Vandaele, S., Nordquist, D. T., Feddersen, R. M., Tretjakoff, I., Peterson, A.C. \& Orr, H. T. (1991). Purkinje cell protein-2 regulatory regions and transgene expression in cerebellar compartments. Genes and Development 5, 1136-1148.

Walker, D. G., Boyes, B. E., McGeer, P. L. \& McGeer, E. G. (1989). Strategies for the identification of novel brain specific genes atfected in Alzheimer disease. Canadian Journal of the Neurological Sciences 16, 483-489.

Watt, D. C. \& Edwards, J. H. (1991). Doubt about evidence for a schizophrenia gene on chromosome 5. Psychological Medicine 21, 279-285.

Weiler, G. H., Sommerfeldt, M., Papandrikopoulou, A., Mischek, U., Bonitz, D., Frey, A., Grupe, M., Scheerer, J. \& Gassen, H. G. (1990). Synthesis of apolipoprotein A-1 in pig brain microvascular endothelial cells. Journal of Neurochemistry $54,444-450$. 